\title{
Adaptive Flow Control of Low Reynolds Number Aerodynamics Using a Dielectric Barrier Discharge Actuator
}

\author{
Young-Chang $\mathrm{Cho}^{* 1}$, Matthew Fledderjohn ${ }^{\dagger 1}$, Matthew Holzel $^{\dagger 1}$, Balaji Jayaraman ${ }^{\ddagger 2}$, \\ Mario Santillo $^{\dagger 1}$, Dennis S. Bernstein ${ }^{\S 1}$, and Wei Shyy ${ }^{* * 1}$ \\ ${ }^{I}$ Department of Aerospace Engineering, University of Michigan, Ann Arbor, MI 48109 \\ ${ }^{2}$ T-3 Fluid Dynamics Group, Los Alamos National Laboratory, NM 87545
}

\begin{abstract}
The aerodynamics of micro air vehicles (operating at the chord Reynolds number of $10^{5} \mathrm{or}$ below) is substantially influenced by the unsteadiness of the wind gust and the aircraft's light weight. In this effort, we investigate the active flow control of the dielectric barrier discharge actuator for flows around the SD7003 airfoil, with the chord Reynolds number of $6 \times 10^{4}$, to enhance our understanding of the unsteady aerodynamics. Using a recently developed ARMARKOV/Toeplitz control scheme, the characteristics of the adaptive control in response to the fluctuation of the free stream, and impact on the aerodynamics are probed. By varying the voltage amplitude to the DBD actuator, effective control of unsteady flow structure can be performed to attain a desirable lift.
\end{abstract}

\section{Nomenclature}

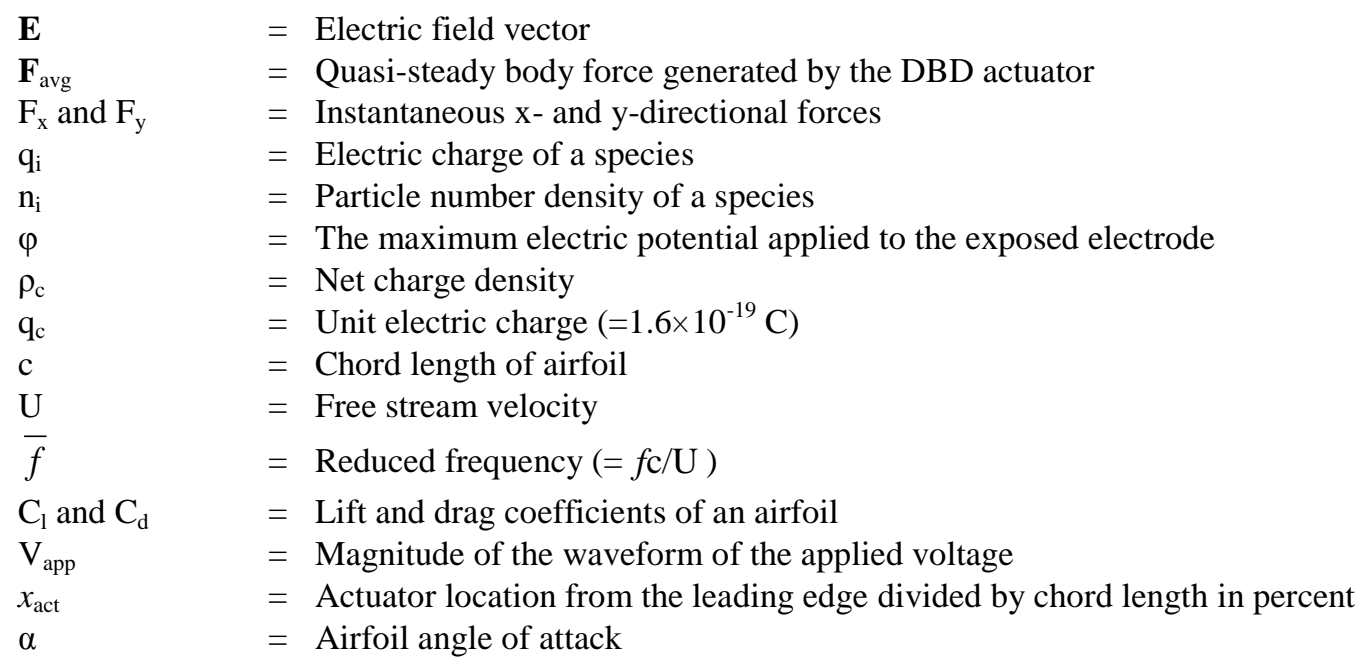

\section{Introduction}

Micro air vehicles (MAVs) have great potential for surveillance and monitoring missions. Such vehicles are usually defined as having dimensions not exceeding $15 \mathrm{~cm}$ in any one direction and maximum flight speeds of

\footnotetext{
* Graduate Research Assistant, Student Member AIAA

${ }^{\dagger}$ Graduate Student

* Technical Staff Member (Research Associate), Member AIAA

${ }^{\S}$ Professor

** Clarence L "Kelly” Johnson Professor, Fellow AIAA
}

American Institute of Aeronautics and Astronautics 
around $10 \mathrm{~m} / \mathrm{s}$. As a consequence, these vehicles operate at Reynolds numbers of less than $10^{5}$. Due to the wind gust and the aircraft's light weight, the aerodynamics of MAVs is substantially influenced by the unsteadiness of the flight environment ${ }^{1}$. Active flow control, while not necessarily applicable in current technological practice, offers a useful way to help examine the fluid physics and issues relevant to vehicle design. In particular, near wall actuation including boundary layer control is interesting scientifically.

Boundary layer control over airfoils has been studied extensively, especially for the low Reynolds number applications. As explained clearly by Lissaman (Ref. 2), a low Reynolds number flow over an airfoil may involve flow separation, transition, and reattachment at the same time. Since the size of the separation bubble is comparable to the airfoil itself, aerodynamic performance of an airfoil is very sensitive to the airfoil shape and flow conditions ${ }^{3}$. The behavior of a laminar separation bubble depending on the angle of attack or Reynolds number can make a dramatic change in the aerodynamic performance, including the lift-to-drag ratio, stall angle and type ${ }^{4}$, and maximum lift. Furthermore, the free stream turbulence intensity and unsteadiness in the flow field are known to affect the transition point, the size of laminar separation bubble, and their unsteady evolution significantly ${ }^{1}$.

The benefits of airfoil flow control devices can be enumerated as the decrease or removal of the laminar separation bubble by momentum injection, the promotion of reattachment through transition to turbulence, and the suppression of large- or small-scale vortex-structure evolution. For low Reynolds number flows with a moderate angle of attack, since the size of the laminar separation bubble dominates the aerodynamics of an airfoil, eliminating the bubble enhances performance considerably. For high angle of attack, however, it is difficult to overcome the severe adverse pressure gradient by only using promoted transition or low-power momentum injection. For example, in Ref. 5 for the low or modest angle of attack SD7003 airfoil, laminar separation bubble and the subsequent turbulent flow region are removed or decreased dramatically with a single actuator. At angles of attack higher than $15^{\circ}$, however, a single actuator near the leading edge cannot induce a significant difference in separation and transition points. By using multiple actuators on the upper surface of the airfoil the injected momentum results in the state close to reattachment at some time instant, but the high angle of attack case shows instability of the separated structure, and as a result significant fluctuation of aerodynamic coefficients. For high angles of attack, the counterflow actuation ${ }^{5}$ as well as unsteady forcing ${ }^{6}$ is reported to be more efficient for facilitating flow reattachment.

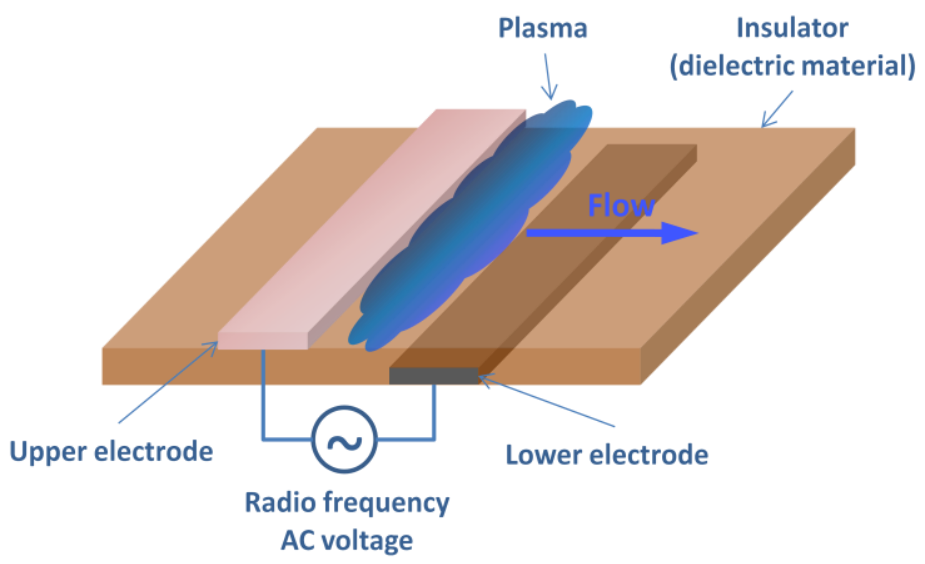

Figure 1. Dielectric-barrier discharge configuration.

The dielectric-barrier discharge (DBD) actuator has been drawing attention as a flow control device ${ }^{7}$. It is comprised of two asymmetrically placed electrodes separated by a dielectric barrier (insulator) and driven by the kilohertz radio frequency AC or pulses with kilo-volt amplitude as shown in Figure 1. The device has neither any components for mass injection nor mechanical moving parts. It has been known that the gas discharge generates a weakly ionized gas, and charged particles under the time-varying electric field generate neutral fluid flow via momentum coupling ${ }^{8,9}$. Asymmetric geometry and charged particle dynamics ${ }^{10,11}$, as well as disparity in discharges of two half-cycles ${ }^{12,13}$ are suggested to explain the resulting uni-directional flow generation.

Despite these efforts to accurately capture the actuator physics, a significant difference in plasma and neutral flow time scales at low Reynolds numbers ${ }^{14}$ makes the numerical approaches inefficient and infeasible for most practical problems. In order to significantly decrease the computational cost, instead of using time-variant high fidelity discharge models, a simplified phenomenological model representation is proposed ${ }^{15}$ to approximate average 
body force field. This enables the simulation of complex flow fields at much smaller computational cost than first principle based models. This approach has been applied to the flow control in low Reynolds number airfoil ${ }^{5,16}$, lowpressure turbine ${ }^{17}$, yielding separation elimination or delay.

Because of its capability of both steady and unsteady actuation up to a high operational frequency (several $\mathrm{kHz}$ ), the DBD actuator can be a versatile control device for various applications. Depending on the flow and flight conditions, the duty cycle - the time duration that the actuator is turned on -or the amplitude of the applied voltage can be adjusted to accommodate performance and power requirements. The efficiency in separated flow control, which depends on operational parameters such as duty cycle and frequency, number of actuators and position, and voltage amplitude, has been focused on by many researchers. Goksel et al. report a reduced duty frequency close to 1 maximizes lift of the airfoil Eppler E338 for a massively separated flow, and between 0.4 and 0.6 for a thin plate ${ }^{18}$. To increase the effects on the wake behind circular cylinder Asghar et al. suggest to locate the actuator near $\pm 90^{\circ}$ points where the average flow separation occurs ${ }^{19}$. Huang et al. employ a closed-loop PID controller based on the variable structure model to increase the power efficiency in noise reduction using DBD actuators ${ }^{20}$.

On the other hand, the flight environments especially at low Reynolds numbers are highly susceptible to gusty conditions $^{1}$. For example, the sinusoidal variation in free stream velocity results in instantaneous aerodynamic performance much different from the steady free stream condition, associated with the unsteady transition position and hysteresis in force variation cycle ${ }^{21}$. It is critical for low Reynolds number flyers not only to obtain favorable aerodynamic performance but also to be capable of rejecting the influence of disturbances. In this context, an efficient control scheme combined with responsive flow actuation device is indispensable to low Reynolds number flyers.

The purpose of feedback control is to cope with dynamic performance changes and enable responsive and robust control, which may not be feasible by either using steady actuation or open loop control. For example, modelbased closed loop control approaches use reduced order models and apply standard control techniques such as linear quadratic regulator (LQR) combined with an observer to reconstruct unknown information ${ }^{22,23}$. Since flow structures at low Reynolds number are complex systems with unsteady flow dynamics, a model-based control schemes need either a dedicated analytic model for the full-state information or a reliable observer design.

The approach adopted in this study is a minimal modeling adaptive control scheme. Markov parameters and moving average coefficients of the flow system are estimated by using the time-domain system identification algorithm based on ARMARKOV/Toeplitz models. A retrospective performance cost function ${ }^{24}$ is defined and minimized to settle the performance to a command or mitigate the fluctuations in aerodynamic coefficients. An adaptive algorithm essentially regulates controller parameters in run-time by tuning the parameters via performance optimization $^{25}$. Compared to the model-based control schemes, the current approach dispenses with detailed understanding on the system, and sets unknown parameters by the adaptive algorithm ${ }^{26,27}$.

System identification and disturbance rejection based on ARMARKOV/Toeplitz algorithm are effectively applied to a 2-dimensional channel flow control $^{27}$ and a separated flow control on a NACA 0025 airfoil using synthetic jet actuators ${ }^{28}$. In this experimental study Tian et al. set the performance variable as the pressure fluctuations on the airfoil surface, and show that the lift-to-drag ratio can be improved by more than 5 for a massively separated flow by using multiple actuators.

In this study an adaptive control scheme is used to explore an efficient and robust control method of low Reynolds number aerodynamics by applying a DBD actuator. As a first step to developing an efficient control strategy, the attached or moderately separated flow over the low Reynolds number airfoil, SD7003 is focused on. Reynolds number based on the airfoil chord length is set to 60,000.

\section{Numerical Approaches}

\section{A. Flow Solver and DBD Actuator Model}

The flow field is analyzed by solving the Reynolds-averaged Navier-Stokes equations using the pressure-based solver adopting the generalized semi-implicit method for pressure-linked equations (SIMPLE) for three-dimensional curvilinear coordinates ${ }^{5}$. For the turbulence closure Wilcox's k- $\omega$ turbulence model is used; for simplicity, the transition model is not applied to this study. The coefficients and boundary conditions for the turbulence model can be referred in Ref. 5. Since the states of ion and electron are non-equilibrium and the ion temperature is comparable to the neutral fluid, the neutral fluid is treated as being isothermal. Considering the time scale disparity between the flow and the radio frequency (RF) actuator operation, the force acting on the neutral fluid is assumed to be a quasi-

3

American Institute of Aeronautics and Astronautics 
steady body force. The body force felt by the neutral flow is equivalent to the Lorentz force acting on the net charge density. For the unsteady operation of the actuator only the amplitude variation of the operation voltage with time scales much larger than the RF operation is considered. The relevant conservation equations are

$$
\begin{gathered}
\frac{\partial \bar{A}}{\partial t}+\frac{\partial \bar{B}}{\partial x}+\frac{\partial \bar{C}}{\partial y}=\bar{D} \\
\text { where } \bar{A}=\left[\begin{array}{c}
\rho \\
\rho u \\
\rho v \\
e
\end{array}\right], \bar{B}=\left[\begin{array}{c}
\rho u \\
\rho u^{2}+p-\tau_{x x} \\
\rho u v-\tau_{x y} \\
(e+p) u-\tau_{x x} u-\tau_{x y} v-k \partial_{x} T
\end{array}\right], \bar{C}=\left[\begin{array}{c}
\rho v \\
\rho u v-\tau_{x y} \\
\rho v^{2}+p-\tau_{y y} \\
F_{x}=E_{x} \sum_{k} q_{k} n_{k}, \quad F_{y}=E_{y} \sum_{k} q_{k} n_{k} .
\end{array} \text {, and } \bar{D}=\left[\begin{array}{c}
0 \\
F_{x} \\
F_{y} \\
0
\end{array}\right] .\right.
\end{gathered}
$$

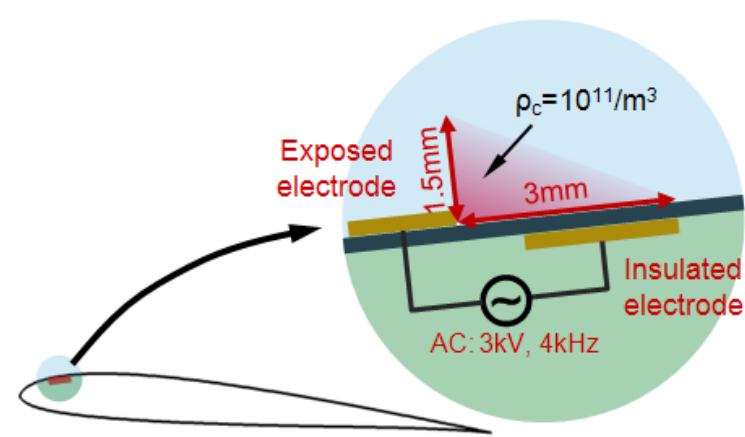

Figure 2. Simplified DBD model geometry.

The DBD actuator model is a simplified model with linear electric field and constant net charge density ${ }^{5,15}$. As presented in Figure 2, this model prescribes localized body forces in a triangular plasma region bounded by two electrodes and dielectric surface. The electric field distribution inside the plasma region is approximated by a spatially linear relation as

$$
|\mathbf{E}|=E_{0}(t)-k_{1} x-k_{2} y,
$$

where $E_{0}(t)=\frac{\phi(t)}{d}, E_{x}=\frac{|\mathbf{E}| k_{2}}{\sqrt{k_{1}^{2}+k_{2}^{2}}}$, and $E_{y}=\frac{|\mathbf{E}| k_{1}}{\sqrt{k_{1}^{2}+k_{2}^{2}}}$. This is a solution of Gauss' equation with the constant net charge density assumption. In (3) the maximum electric field intensity, $\mathrm{E}_{0}$ is defined based on the applied voltage amplitude, and $k_{1}$ and $k_{2}$, the slopes of the electric field attenuation away from the exposed electrode and dielectric surface are set to allow the breakdown voltage at the boundary with the minimum electric field strength. This analytical-empirical model represents a time-averaged body force component acting on the fluid as ${ }^{5}$

$$
\mathbf{F}_{\text {avg }}(x, y, t)=\rho_{c} q_{c} \delta \frac{\Delta t}{T} \mathbf{E}(x, y, t) .
$$

Since the constant charge density $\rho_{\mathrm{c}}$ with unit charge $q_{\mathrm{c}}$ is present only inside the plasma region, $\delta$ is set to 0 or 1 depending on the position. The discharge duty cycle is the portion of time at which effective force generation is occurring in each operation cycle. For the purpose of the control input the applied voltage to the electrode is changed depending on the controller command, resulting in a time variable body force. Since the simplified DBD model is based on the quasi-steady assumption using the time scale disparity, the unsteady control input is meaningful when its timescale lies between those of low Reynolds number flow and plasma operation. The current simplified DBD model presents good agreement with experimental data in terms of force generation ${ }^{11}$ and maximum induced flow velocity ${ }^{29}$.

In order to assess the resultant performance of the DBD actuator and controller, a single co-flow directional DBD actuator with voltage amplitude modulation according to the controller output is used, and the position is fixed to be either $3.5 \%$ or $5 \%$ from the airfoil leading edge in this study. 


\section{B. ARMARKOV/Toeplitz Models}

The transfer function of a $n$-th order discrete-time finite dimensional linear time-invariant system can be represented as

$$
G(z)=\frac{B_{0} z^{n}+B_{1} z^{n-1}+\cdots+B_{n}}{z^{n}+a_{1} z^{n-1}+\cdots+a_{n}}
$$

Using a non-minimal basis, the system output $y(k)$ at sample $k$ can be expressed explicitly as a function of the past output, and the past and current control input $u$ as

$$
y(k)=\sum_{j=1}^{n}-\alpha_{j} y(k-\mu-j+1)+\sum_{j=1}^{\mu} H_{j-1} u(k-j+1)+\sum_{j=1}^{n} B_{j} u(k-\mu-j+1),
$$

where $\alpha_{j}$ and $B_{, j}$ can be expressed using $a_{k}$ and $B_{k}$ in (5) as in Ref. 24, and $H_{j}$ 's are the Markov parameters of the system. Since (6) is represented with $\mu$-Markov parameters, it provides more flexibility in identifying the system by allowing direct estimation of those parameters ${ }^{30}$.

The adaptive feedback control system in the current application is presented in Figure 3. The system output $\Delta \mathrm{C}_{1}$ is the difference between the measured and the nominal lift coefficient $C_{10}$ which is the lift coefficient corresponding to the nominal voltage $\mathrm{V}_{0}$. The performance variable $z$ is defined as the difference between the current and target lift increments $\Delta \mathrm{C}_{\mathrm{lc}}$ and $\Delta \mathrm{C}_{1}$ respectively. The control output $u$ is the applied voltage $\mathrm{V}_{\text {app }}$ minus the nominal voltage $\mathrm{V}_{0}$.

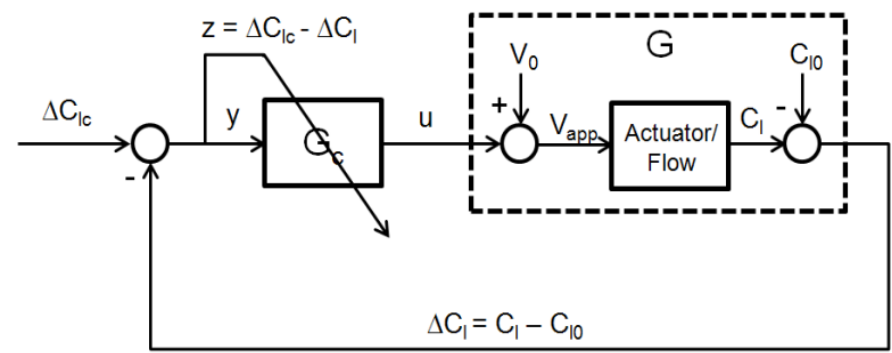

Figure 3. Block diagram of the system and adaptive controller.

For a standard two-input two-output system, the control output $u$ and noise $w$ that can be substituted by the target lift increment $\Delta \mathrm{C}_{\mathrm{lc}}$, and corresponding transfer matrices $G_{z_{u, u}}$ and $G_{z_{w}, w}$ are used to express the performance. Using the ARMARKOV model, the performance can be expressed as

$$
\begin{aligned}
z(k) & =z_{w}(k)+z_{u}(k) \\
& =G_{z_{w} w} w(k)+G_{z_{u},} u(k) \\
& =\Delta C_{l c}+\sum_{j=1}^{\mu} H_{z u, j-1} u(k-j+1)+\sum_{j=1}^{n} B_{z u, j} u(k-\mu-j+1) .
\end{aligned}
$$

Furthermore, the extended performance vector $Z(k)$ and the extended control vector $U(k)$ can be defined as

$$
Z(k)=\left[\begin{array}{c}
z(k) \\
\vdots \\
z(k-p+1)
\end{array}\right], \quad U(k)=\left[\begin{array}{c}
u(k) \\
\vdots \\
u(k-\mu-n-p+2)
\end{array}\right] .
$$

Then the model relating the performance history, the target lift increment and control input can be expressed in the form as

$$
\begin{aligned}
Z(k) & =Z_{w}(k)+Z_{u}(k) \\
& =G_{z_{w} w} w(k)+G_{z_{u} u} U(k) \\
& =\Delta C_{l c} I_{p}+B_{z u} U(k),
\end{aligned}
$$


where

$$
B_{z u}=\left[\begin{array}{ccccccccc}
H_{z u, 0} & \cdots & H_{z u, \mu-1} & B_{z u, 1} & \cdots & B_{z u, n} & 0 & \cdots & 0 \\
0 & \ddots & & \ddots & \ddots & & \ddots & \ddots & \vdots \\
\vdots & \ddots & \ddots & & \ddots & \ddots & & \ddots & 0 \\
0 & \cdots & 0 & H_{z u, 0} & \cdots & H_{z u, \mu-1} & B_{z u, 1} & \cdots & B_{z u, n}
\end{array}\right] .
$$

The block-Toeplitz ARMARKOV control matrix $B_{z u} \in R^{p \times(\mu+n+p-1)}$ consists of system parameters including $\mu$ Markov parameters and needs to be identified.

On the other hand, the control output can be represented as

$$
\begin{aligned}
u(k)= & \sum_{j=1}^{n_{c}}-\alpha_{c, j}(k) u\left(k-\mu_{c}-j+1\right)+\sum_{j=1}^{\mu_{c}} H_{c, j-1}(k) y(k-j+1) \\
& +\sum_{j=1}^{n_{c}} B_{c, j}(k) y\left(k-\mu_{c}-j+1\right)
\end{aligned}
$$

And using the control regressor vector $\Phi_{u y}(k)$ and the controller parameter vector $\theta(k) \in R^{1 \times\left(2 n_{c}+\mu_{c}-1\right)}$, the control output can be given by ${ }^{24}$

$$
\begin{aligned}
& u(k)=\theta(k) R_{1} \Phi_{u y}(k), \\
& \text { and thus } U(k)=\sum_{i=1}^{p_{c}} L_{i} \theta(k-i+1) R_{i} \Phi_{u y}(k),
\end{aligned}
$$

where

$$
\begin{aligned}
& \theta(k) \triangleq\left[\begin{array}{lllllllll}
-\alpha_{c, 1}(k) & \cdots & -\alpha_{c, n_{c}}(k) & H_{c, 0}(k) & \cdots & H_{c, \mu_{c}-1}(k) & B_{c, 1(k)} & \cdots & B_{c, n_{c}(k)}
\end{array}\right], \\
& \Phi_{u y}(k) \triangleq\left[\begin{array}{c}
u\left(k-\mu_{c}\right) \\
\vdots \\
u\left(k-\mu_{c}-p_{c}-n_{c}+2\right) \\
y(k-1) \\
\vdots \\
y\left(k-\mu_{c}-p_{c}-n_{c}+2\right)
\end{array}\right], \\
& L_{i}(k) \triangleq\left[\begin{array}{c}
0_{(i-1) \times 1} \\
1 \\
0_{\left(p_{c}-i\right) \times 1}
\end{array}\right] \text {, } \\
& R_{i} \triangleq\left[\begin{array}{cccccc}
0_{n_{c} \times(i-1)} & I_{n_{c} \times n_{c}} & 0_{n_{c} \times\left(p_{c}-i\right)} & 0_{n_{c} \times(i-1)} & 0_{n_{c} \times\left(n_{c}+\mu_{c}-1\right)} & 0_{n_{c} \times\left(p_{c}-i\right)} \\
0_{\left(n_{c}+\mu_{c}-1\right) \times(i-1)} & 0_{\left(n_{c}+\mu_{c}-1\right) \times n_{c}} & 0_{\left(n_{c}+\mu_{c}-1\right) \times\left(p_{c}-i\right)} & 0_{\left(n_{c}+\mu_{c}-1\right) \times(i-1)} & I_{\left(n_{c}+\mu_{c}-1\right) \times\left(n_{c}+\mu_{c}-1\right)} & 0_{\left(n_{c}+\mu_{c}-1\right) \times\left(p_{c}-i\right)}
\end{array}\right] .
\end{aligned}
$$

Since the adaptive control output is based on optimizing the control parameters in accordance with the given performance, a cost function of the performance is defined and minimized. By using $k$-th controller parameter $\theta(k)$ instead of $\theta(k-i+1)$, the retrospective performance and its cost function can be defined as

$$
\begin{aligned}
& \hat{Z}(k) \triangleq \Delta C_{l c} I_{p}+B_{z u} \sum_{i=1}^{p_{c}} L_{i} \theta(k) R_{i} \Phi_{u y}(k), \\
& J(k) \triangleq \frac{1}{2} \hat{Z}^{T}(k) \hat{Z}(k) .
\end{aligned}
$$

Updating the controller parameter can be done by finding the controller parameter that minimizes the cost function 


$$
\theta(k+1) \triangleq \theta(k)-\eta(k) \frac{\partial J(k)}{\partial \theta(k)}, \quad \text { where } \frac{\partial J(k)}{\partial \theta(k)}=\sum_{i=1}^{p_{e}} L_{i}^{T} B_{z u}^{T} \hat{Z}(k) \Phi_{u y}^{T}(k) R_{i}^{T} .
$$

The adaptive step size is implemented as

$$
\eta(k) \triangleq \frac{1}{p_{c}\left\|B_{z u}(k)\right\|_{F}^{2}\left\|\Phi_{u y}(k)\right\|_{2}^{2}}
$$

The update law (18) guarantees to decrease the performance error $\varepsilon(\mathrm{k})$, the difference between the current performance and the desired performance ${ }^{24}$

$$
\begin{aligned}
\varepsilon(k) & \triangleq \hat{Z}^{*}(k)-\hat{Z}(k) \\
& =B_{z u}\left(\sum_{i=1}^{p_{c}} L_{i} \theta^{*} R_{i} \Phi_{u y}(k)-\sum_{i=1}^{p_{c}} L_{i} \theta(k) R_{i} \Phi_{u y}(k)\right),
\end{aligned}
$$

where the desired performance is assumed to have the controller parameter $\theta^{*}$ which minimizes the cost function for all $\mathrm{k}$.

Several features can be mentioned in this formulation. The matrix $B_{z u}$ needs to be identified prior to the controller operation. In the system identification stage the order of the controller, $n_{c}$ is chosen carefully to maximize the transient performance. The singular values of the Markov block Hankel matrix identified using methods such as $\mathrm{OKID}^{31}$ are used as an indicator of the order of the system. Although a sharp drop of the singular values at the order higher than the true order of the system is observed in a perfectly linear system, a gradual decrease is typical in nonlinear system. In addition, the amplitude of the signal for system identification purposes should not excite the system too far away from the desired equilibrium, while it should contain enough frequency content and magnitude to excite all the system modes of interest.

\section{Recursive Least Squares (RLS) System Identification}

In order to obtain the Markov Parameters and the $B_{z u}$ matrix of the system required for the controller, the DBD actuator is excited by a known white noise signal as the voltage input. The input and the resulting lift coefficient are used in the recursive least squares (RLS) algorithm ${ }^{32,33}$ to identify the system parameters.

Regarding the coefficients of the time series as the unknown values, (6) can be expressed as

$$
\theta \Phi_{u y}=Y,
$$

where

$$
\begin{aligned}
& \theta=\left[\begin{array}{lllllllll}
H_{0} & \cdots & H_{\mu-1} & B_{1} & \cdots & B_{n} & -\alpha_{1} & \cdots & -\alpha_{n}
\end{array}\right] \text {, } \\
& \begin{aligned}
\Phi_{u y} & =\left[\begin{array}{ccc}
u(\mu+n) & \cdots & u(\mu+n+i-1) \\
\vdots & \vdots & \vdots \\
u(1) & \cdots & u(i) \\
y(n) & \cdots & y(n+i-1) \\
\vdots & \vdots & \vdots \\
y(1) & \cdots & y(i)
\end{array}\right], \\
Y & =\left[\begin{array}{lll}
y(\mu+n) & \cdots & y(\mu+n+i-1)
\end{array}\right] .
\end{aligned}
\end{aligned}
$$

Here $\mu+n+i-1$ is the total number of measurements obtained from the white noise excitation and $\theta \in R^{1 \times(2 n+\mu)}$ is the unknown parameter vector, $\Phi_{u y} \in R^{(2 n+\mu) \times i}$ is the regressor matrix, and $Y \in R^{p \times i}$ is the output vector. RLS finds the system parameter $\hat{\theta}$ that minimizes $J(x)$ in the least squares problem

$$
J(x)=\left(\hat{\theta} \Phi_{u y}-Y\right)^{T}\left(\hat{\theta} \Phi_{u y}-Y\right) .
$$

The exact solution requires taking the inverse of $\Phi_{i} \Phi_{i}^{T}$ at each time step, which may be numerically ill-conditioned. The solution to the least squares problem can be solved recursively by updating the estimated $\hat{\theta}_{i}$ with new input and 
output data at each time step. The following, equivalent and more convenient, RLS algorithm does not require a matrix inversion. Let

$$
\hat{\theta}_{i+1}=\hat{\theta}_{i}+\left(y_{i+1}-\hat{\theta}_{i} \Phi_{i+1}^{\prime}\right) L
$$

where $y_{i+1}$ is the additional output measurement and $\Phi_{i+1}^{\prime} \in R^{(2 n+\mu) \times 1}$ is the additional input and output measurement vector and $L$ is given by

$$
\begin{aligned}
& L=\frac{1}{1+\Phi_{i+1}^{\prime T} P_{i} \Phi_{i+1}^{\prime}} \Phi_{i+1}^{\prime T} P_{i}, \\
& P_{i+1}=P_{i}\left(I-\Phi_{i+1}^{\prime} L\right) .
\end{aligned}
$$

The algorithm requires initial conditions $\hat{\theta}_{0}$ and $P_{0}$, which is equivalent to the least squares estimation when it is initialized to $P_{0}=\Phi_{u y} \Phi_{u y}^{T}$ and $\hat{\theta}_{0}=Y \Phi_{u y}^{T}\left(\Phi_{u y} \Phi_{u y}^{T}\right)^{-1}$ for an invertible $\Phi_{u y} \Phi_{u y}^{T}$. Here we initialize at time $t=0$ with $\hat{\theta}_{0}=0_{1 \times(2 n+\mu)}$ and $P_{0}=c I$ where $\mathrm{c}$ is a large constant. The estimate is equivalent to the batch solution as described in Ref. 32, and given by

$$
\hat{\theta}=\left[\left(\frac{1}{c}\right) \hat{\theta}_{0}+Y \Phi_{u y}^{T}\right]\left[\left(\frac{1}{c}\right) I+\Phi_{u y} \Phi_{u y}^{T}\right]^{-1} .
$$

With a proper choice of the initialization, the recursive algorithm identifies the system parameters without calculating the inverse of the regressor matrix.

\section{Results and Discussion}

\section{A. Open-Loop Control: Steady and Unsteady Actuation}

In order to select the operational conditions for closed-loop control, the effective regime of actuation is surveyed using both constant and time-varying actuations. As concluded in Ref. 5, the constant operation of the DBD actuator increases the lift-to-drag ratio by preventing flow separation for low angle of attack cases. At angles of attack less than $12^{\circ}$ the actuator located at $5 \%$ from the leading edge with a steady $3 \mathrm{kV}$ actuation can efficiently suppress the separated flow region.

However, the unsteady evolution of aerodynamic forces is initiated by the same actuation at high angle of attack, for example $\alpha=15^{\circ}$ as presented in Figure 4. Although the average L/D is much larger than the case without actuation, whose $\mathrm{L} / \mathrm{D}$ is about 3 and wake is stable, the periodic force history decreases the usefulness of the flow actuation. The periodic unsteadiness is mostly related to the vortex evolution on the airfoil initiated by the actuation. From the flow snapshots in Figure 5, it can be identified that a vortex downstream of the actuator is initiated and shed with an unstable flow structure, resulting in a significant change in aerodynamic force. In Figure 5 a) $\sim$ d), a cluster of vortex structures evolves and sheds periodically, and the relevant reduced frequency or Strouhal number $\bar{f}=S t=f c / U$ has the value 0.21 .

The unsteady operation of the DBD actuator, on the other hand, also leads to the time-varying aerodynamic force generation. Since the time scale of vortex evolution is much larger than the plasma operation time scale, which is approximated as a quasi-steady operation, the time-varying modulation in the voltage amplitude is reasonably valid for the simplified DBD model. A square wave signal with $\bar{f}=1.14$ as the input voltage magnitude is applied to the actuator, and the time histories of lift and drag coefficients are compared in Figure 6. For relatively low magnitude of $0-3 \mathrm{kV}$ in Figure $6 \mathrm{a}$ ), there are fluctuations in force with the time scales corresponding to the applied wave as well as the vortex evolution whose reduced frequency is about 0.29 . As the minimum voltage increases to 3 $\mathrm{kV}$ with the wave amplitude of $2 \mathrm{kV}$, a distinct waveform appears with the same reduced frequency as the case with a $3-\mathrm{kV}$ constant actuation.

The open-loop control result with the selected operating parameters provides insight into applying the closedloop controller to the problem. The flow unsteadiness of concern is caused not only by the disturbance from the exterior but also by the inherent flow instability in high angle of attack airfoils. The control authority utilizing the DBD actuator is determined by the dominance of the momentum injection due to the actuator over the unsteady 
vortex transport and evolution mechanism. Considering that, the effective closed-loop control of the flow field using the DBD actuator needs to be based on the categorization depending on the operational conditions such as the angle of attack, the actuation amplitude and the actuator position.

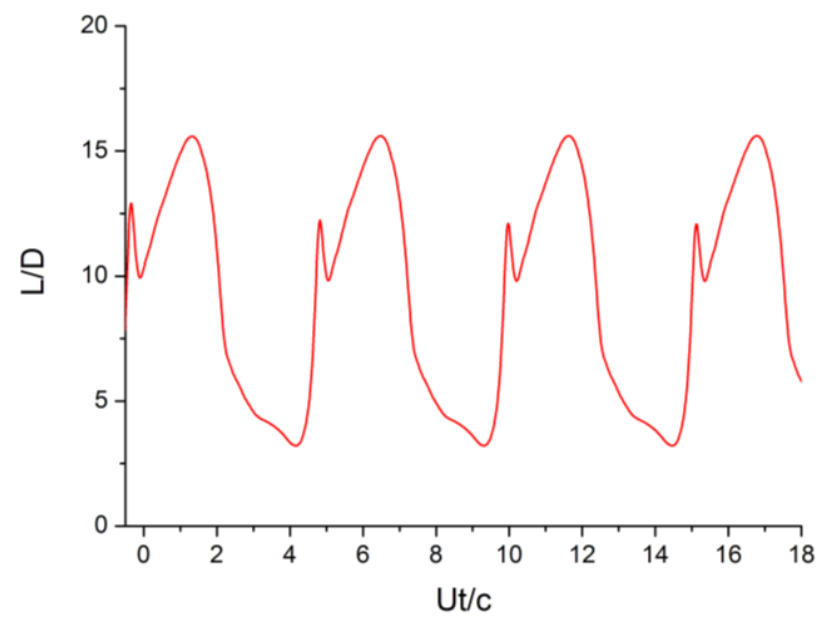

Figure 4. Lift-to-drag ratio time history with $3 \mathrm{kV}$ constant actuation $\left(\alpha=15^{\circ}\right)$.

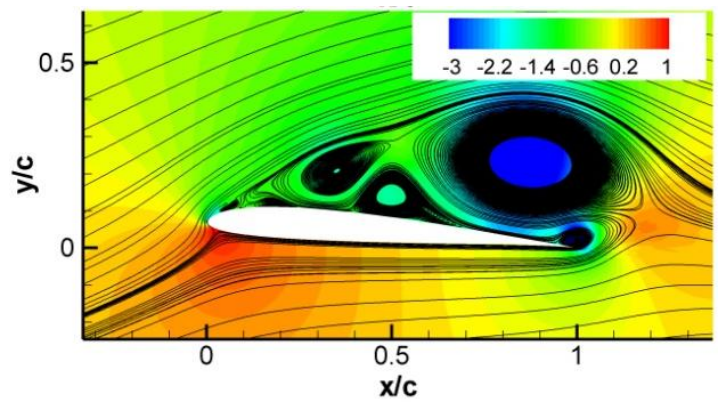

a) $\mathrm{Ut} / \mathrm{c}=3.9$

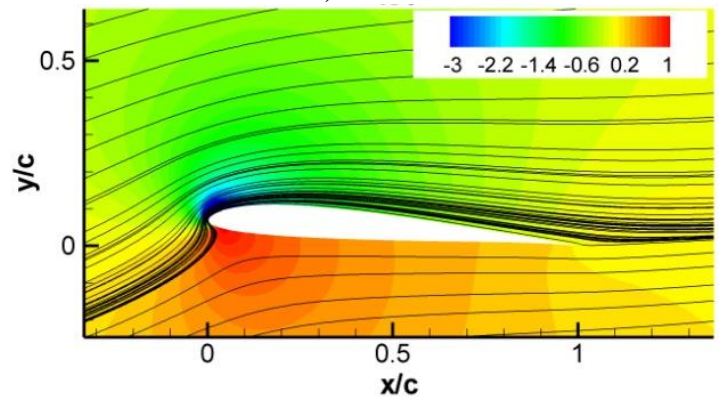

c) $\mathrm{Ut} / \mathrm{c}=11.8$

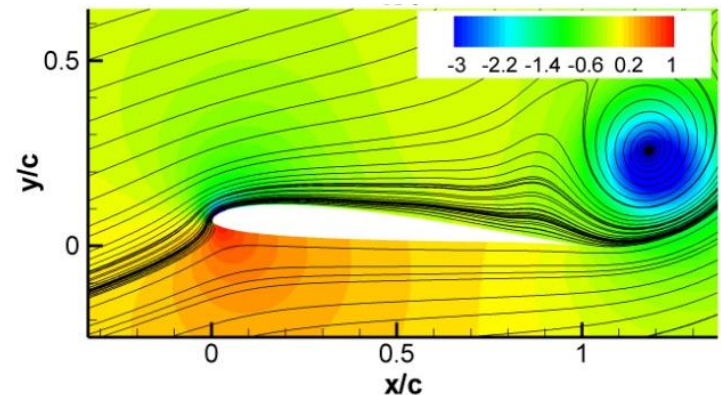

b) $\mathrm{Ut} / \mathrm{c}=5.0$

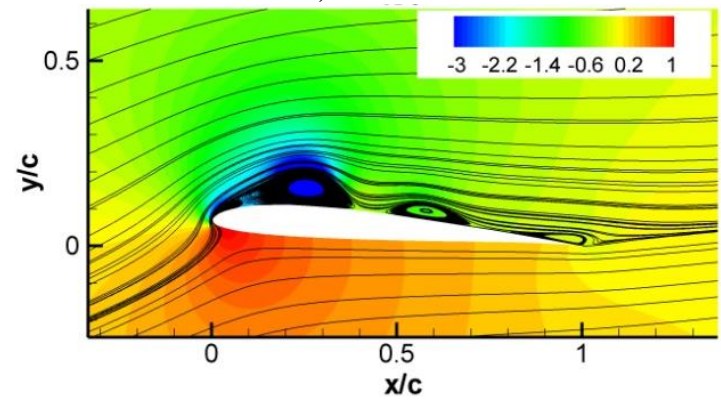

d) $\mathrm{Ut} / \mathrm{c}=13.2$

Figure 5. Vortex evolution in the wake region with $3 \mathrm{kV}$ constant actuation $\left(\alpha=15^{\circ}\right)$ : streamlines and pressure coefficient contours.

The corresponding snapshots of the flow fields are presented in Figure 7. Each snapshot corresponds to the moment when the flow right behind the DBD actuator (marked with a blue circle in the enlarged picture) is accelerated maximally, which does not necessarily coincide with the peak voltage of the square wave. For $\mathrm{V}_{\text {app }}=3-5$ $\mathrm{kV}$, as represented by Figure $7 \mathrm{~b}$ ), the second recirculation is strong enough to sustain the clustered recirculation regions, resulting in a coherent vortex evolution. With the lower voltage magnitude, on the other hand, the flow 
affected by the actuator hardly forms connected recirculation zones, and the vortex evolution is dispersed, resulting in a smaller scale fluctuation in Figure 6 a).

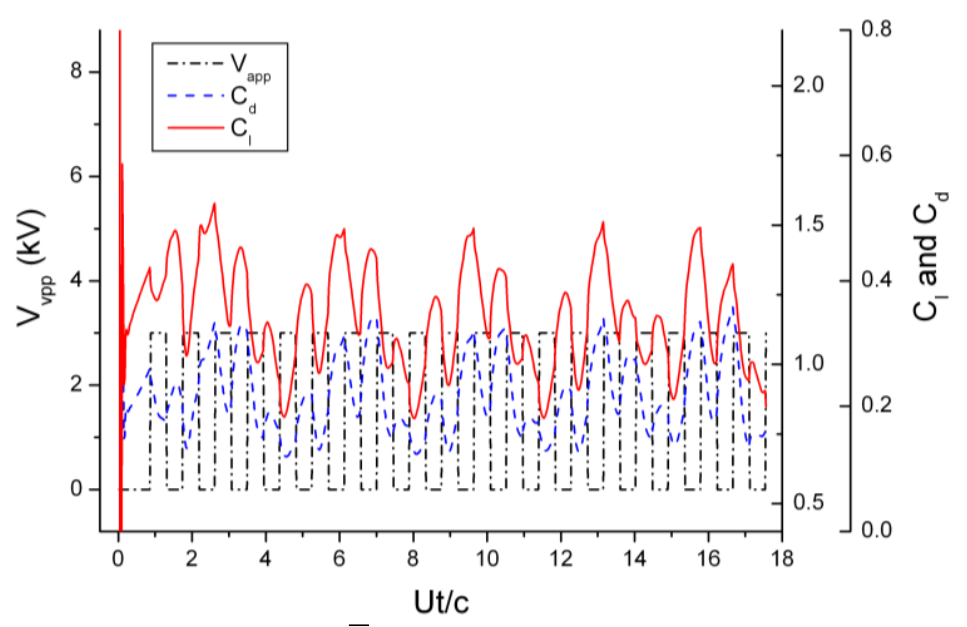

a) $\bar{f}=1.14, \mathrm{~V}_{\text {app }}=0-3 \mathrm{kV}$

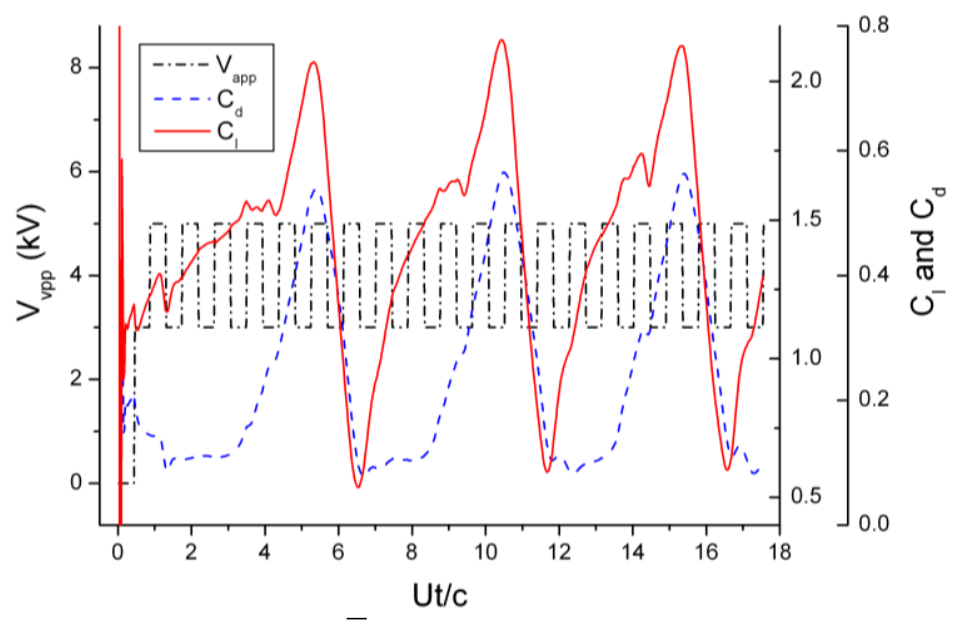

b) $\bar{f}=1.14, \mathrm{~V}_{\text {app }}=3-5 \mathrm{kV}$

Figure 6. Lift and drag coefficients with square-wave actuation $\left(\alpha=15^{\circ}\right)$.

In order to develop an efficient adaptive flow control scheme for low Reynolds number applications, extensive study on the control efficiency for a highly nonlinear flow field is necessary. For this study, as a first step, relatively straightforward cases with low angles of attack accompanying fully attached and moderately separated flow fields over the airfoil are chosen to be controlled. The current effort focuses on assessing the fidelity of system identification and implementing the adaptive control scheme to the low Reynolds number flow/DBD actuator model.

\section{B. Impulse Response and System Identification}

The operational voltage of a DBD actuator a few millimeters in electrode length and operating under the atmospheric pressure is usually several kilovolts. To apply a force variation to the fully attached flow, the voltage range of 3-8 kV is used for the angle of attack $\alpha=2^{\circ}$ as a conventional setup and the actuator is located at $5 \%$ from the leading edge. Flow actuation at a low angle of attack maintaining a separation bubble, however, is not feasible in this voltage range with the DBD actuator model. In order to avoid the vortex-convection dominant flow field at high angle of attack and maintain a stable separation bubble at the same time, the voltage range is adjusted to be 0.1-0.7 $\mathrm{kV}$ for $\alpha=8^{\circ}$ with the actuator placed at 3.5\% from the leading edge. Although this range of voltage is too low to 
induce a gas discharge while maintaining the other conditional parameters, a similarly decreased impact on the flow field can be achieved by adjusting the geometry or duty cycle in practice.

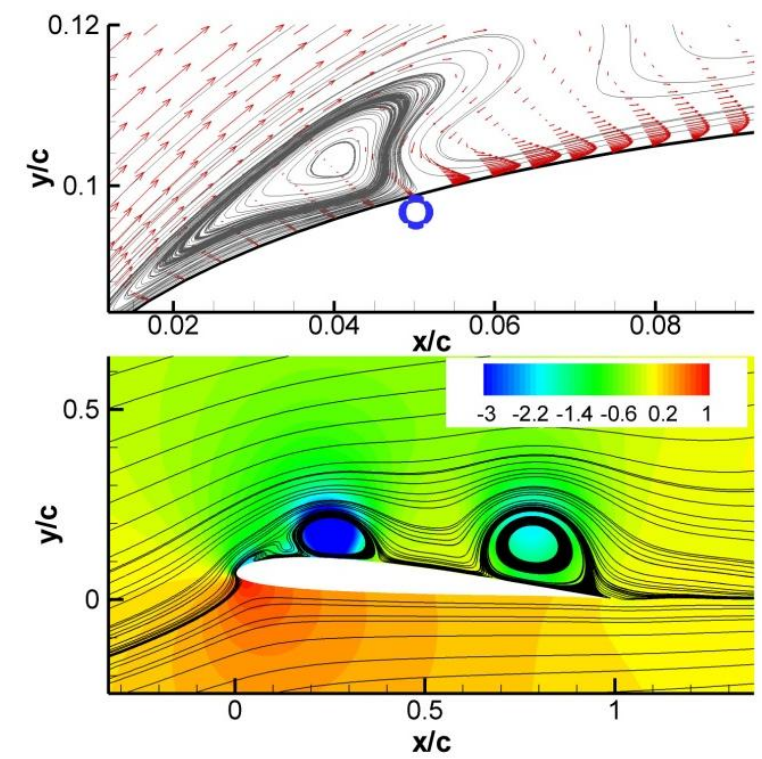

a) $\mathrm{V}_{\text {app }}=0-3 \mathrm{kV}$
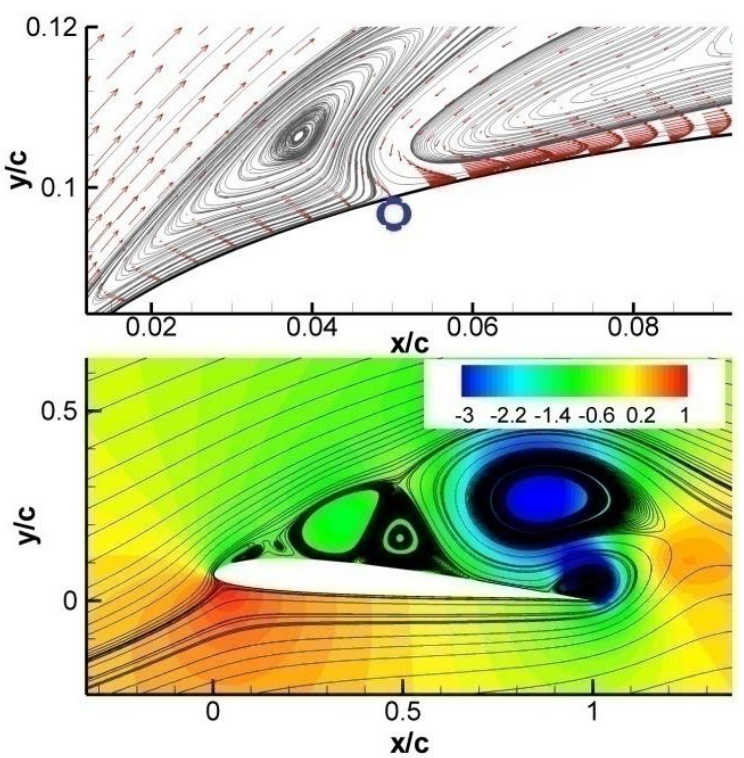

b) $\mathrm{V}_{\text {app }}=3-5 \mathrm{kV}$

Figure 7. Flow field snapshots with velocity vector, streamlines and pressure contours $\left(\alpha=15^{\circ}\right)$.

The flow fields with actuation at the nominal voltages are presented in Figure 8. For the case with $\alpha=2^{\circ}$ the flow is fully attached through the entire input voltage range, and as a result the change in aerodynamic performance is relatively small. For $\alpha=8^{\circ}$, on the other hand, a separation bubble exists on the airfoil, and the performance is more sensitive to the change in the actuation voltage or inlet conditions.

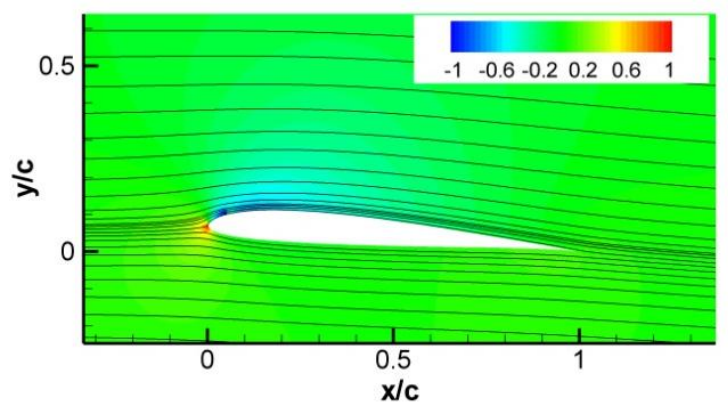

a) $\alpha=2^{\circ}, V_{\text {app }}=5 \mathrm{kV}, x_{\text {act }}=5 \%$

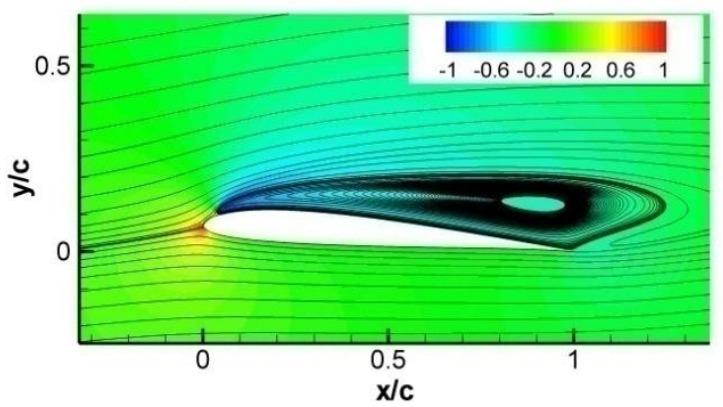

b) $\alpha=8^{\circ}, \mathrm{V}_{\mathrm{app}}=0.4 \mathrm{kV}, \mathrm{x}_{\mathrm{act}}=3.5 \%$

Figure 8. Streamlines and pressure coefficient contours with actuation at the nominal voltages.

The sampling rate of the performance and the update rate of the control output need to be determined based on the time step size of the flow simulation, the convergence criteria of the flow solver with the actuator-induced body force as a source term, and time scale of the flow structure of interest. This study uses a time step size $\Delta \mathrm{t}_{\mathrm{c}}$, which is 10 times larger than the flow simulation time step size $\Delta t$, for the sampling and updating data for the system identification and control purposes. Figure 9 shows the time history of lift coefficient with the impulse actuation, which is applied at $\mathrm{Ut} / \mathrm{c}=0$ with the width of $\Delta \mathrm{t}_{\mathrm{c}}$. The system Markov parameters are identified by sampling $\Delta \mathrm{C}_{\mathrm{l}} / \mathrm{V}_{0}$ $=\left(\mathrm{C}_{1}-\mathrm{C}_{10}\right) / \mathrm{V}_{0}$ with $\Delta \mathrm{t}_{\mathrm{c}}$ as shown in Figure 9. The control/measurement step size is small enough to capture the system response to the relatively low frequency excitation, but large enough to avoid the transient response between the control output updates. 


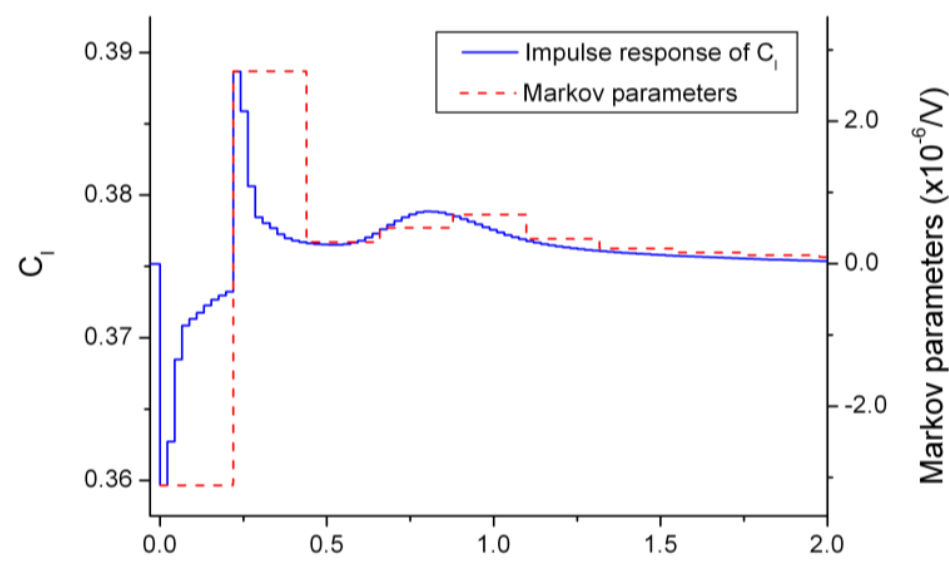

Figure 9. Impulse response of the system and Markov parameters: $\alpha=2^{\circ}, V_{0}=3 \mathrm{kV}$ and $\Delta \mathbf{V}_{\text {app }}=5 \mathrm{kV}$ (without separation bubble).

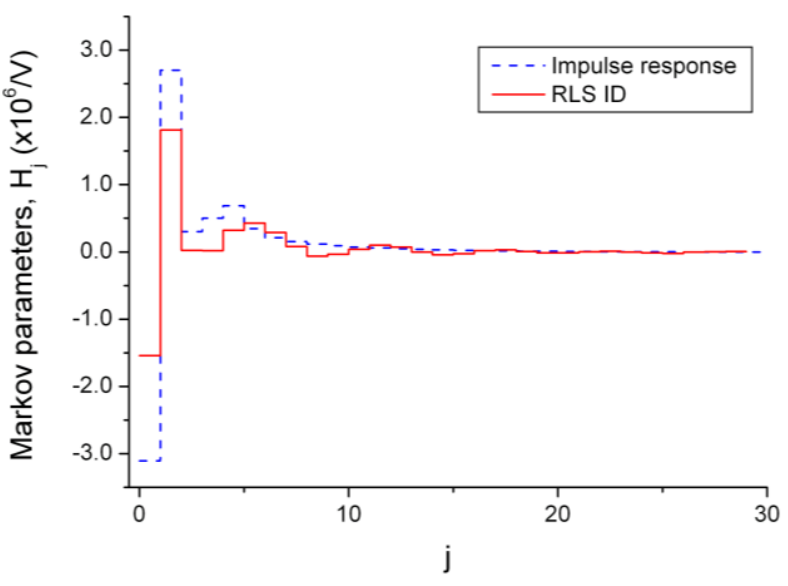

a) $\alpha=2^{\circ}$, without separation bubble

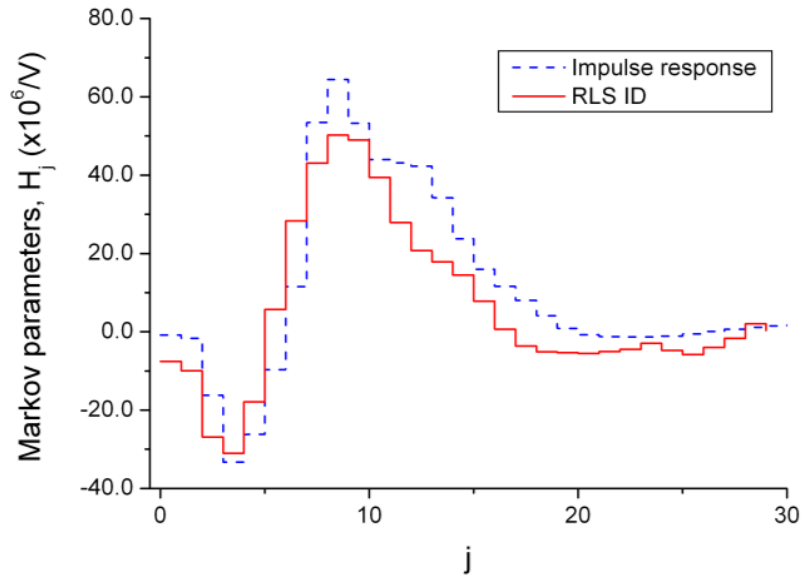

b) $\alpha=8^{\circ}$, with separation bubble

Figure 10. Comparison between the identified Markov parameters and impulse response.

In order to excite the system to identify system parameters, white noise signals band-limited with $12 \mathrm{~Hz}$ cut-off frequency and pertained to the specified voltage ranges, are used as the actuator voltage input. The input and output to the system $\mathrm{G}$ in Figure 3 correspond to $\left(\mathrm{V}_{\text {app }}-\mathrm{V}_{0}\right)$ and $\left(\mathrm{C}_{1}-\mathrm{C}_{10}\right)$, respectively. The $\mu$-Markov model (6) is used for identification, and the number of Markov parameters $\mu$ and the order of the model $n$ are usually set to large enough values to retain the relevant singular values of the system matrix. In the current study $\mu$ and $n$ are set as 25 and 30 , respectively, and it is observed that the identified parameters are not sensitive to $\mu$. In each parameter identification, 500 data points are used, which corresponds to the physical time interval of $U t / c=110$.

For each actuator setup the identified system parameters are compared with the impulse response of the system in Figure 10. Since Markov parameters are identical to the finite impulse response of the discrete-time model, the comparison is a measure of assessing the accuracy of the system identification. For both $\alpha=2^{\circ}$ and $8^{\circ}$ cases the identified Markov parameter sets are reasonably consistent with the impulse responses. The difference between the two cases can be attributed to the different flow dynamics resulting from actuation positions and the existence of flow-separation bubble. Especially, in the case with $\alpha=8^{\circ}$, the sensitivity of output to input is more than 10 times higher than the case with $\alpha=2^{\circ}$.

Using the identified system parameters and the $\mu$-Markov representation, the system output signal due to the white noise input can be reconstructed, and the estimated output is compared with the original output in Figure 11. After the initiation of the discrete-time series model with zero output, the estimated output shows a reasonably good agreement with the original output signal for both cases with $\alpha=2^{\circ}$ and $8^{\circ}$. 

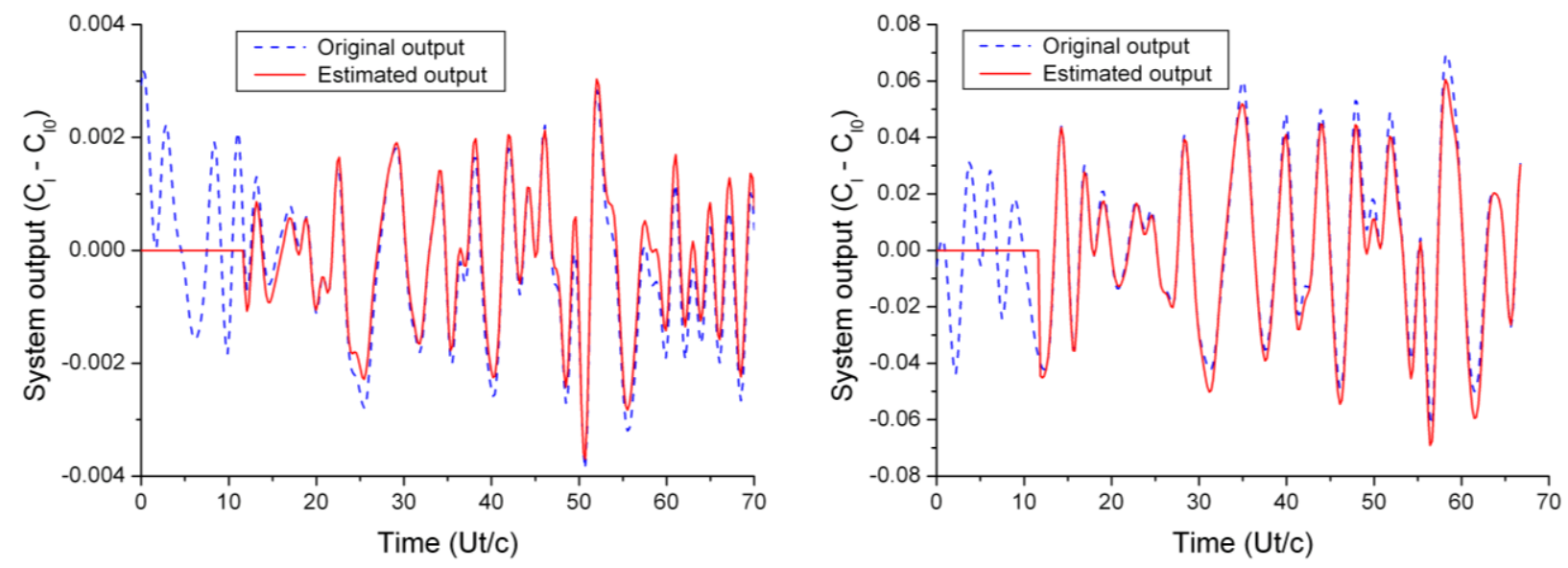

a) $\alpha=2^{\circ}, \mathrm{V}_{\text {app }}=3-8 \mathrm{kV}$ (without separation bubble)

b) $\alpha=8^{\circ}, \mathrm{V}_{\text {app }}=0.1-0.7 \mathrm{kV}$ (with separation bubble)

Figure 11. Comparison between the original signal and the estimated output using RLS ID.

\section{Closed-Loop Actuation with Adaptive Control}

In order to apply the identified parameters to the adaptive feedback controller, lift is controlled according to a command value. The performance variable is defined as the difference between the lift deviation from the nominal value $C_{1}-C_{10}$ and the target lift $\Delta C_{l c}=0.005$ as in Figure 3 and the identified system parameters are used for $\alpha=2^{\circ}$ with other operational conditions. The order of the controller is set as $n_{c}=50$. As presented in Figure 12 the performance converges to zero with the corresponding converged output voltage about $6.8 \mathrm{kV}$. Since the control authority for this fully attached flow is limited, the available target value is too small in a practical sense. However, the result demonstrates the effective contribution of feedback control combined with the flow/DBD actuator model, adjusting the performance in accordance with the command.

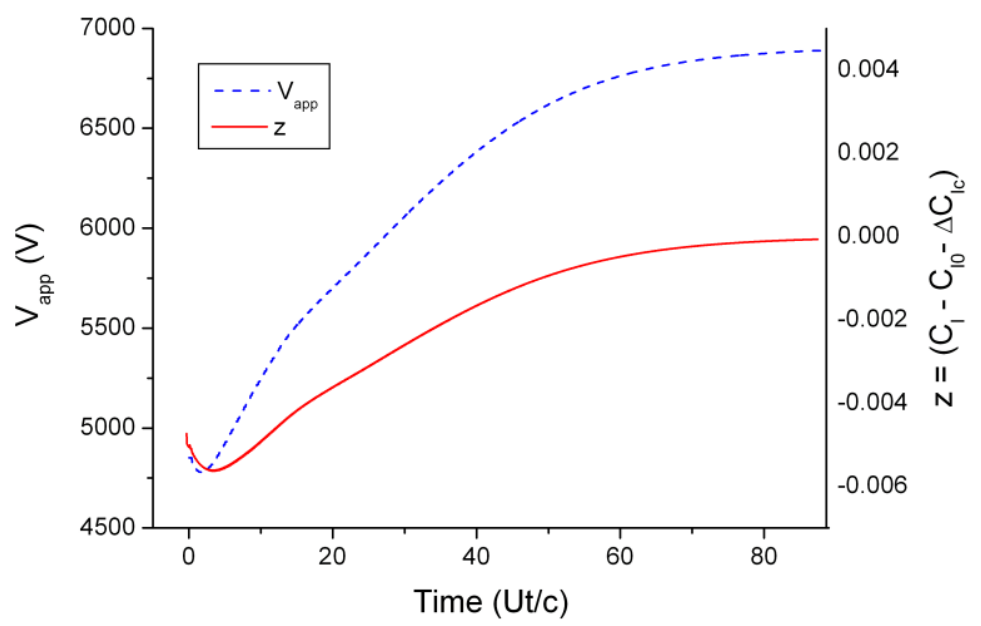

Figure 12. Adaptive control for lift adjustment.

For the next application, a sinusoidal disturbance is added to the inlet velocity and a feedback control with the adaptive controller is performed to regulate the performance. The reduced frequency of the disturbance is 0.137 and the amplitude is $0.5 \%$ of the free stream speed for $\alpha=2^{\circ}$ and $1 \%$ for $\alpha=8^{\circ}$.

In Figure 13 a) the feedback controller is activated at Ut/c $=11.2$ and compensates for the performance fluctuation. It is observed that the output voltage oscillates according to the impact of the disturbance, and the voltage magnitude is increased and constrained by the lower limit $3 \mathrm{kV}$ at some instants. Although the controller 
eventually decreases the performance fluctuation to $20 \%$ of the uncontrolled value, the effect of the inlet disturbance is not completely eliminated within the simulation time.

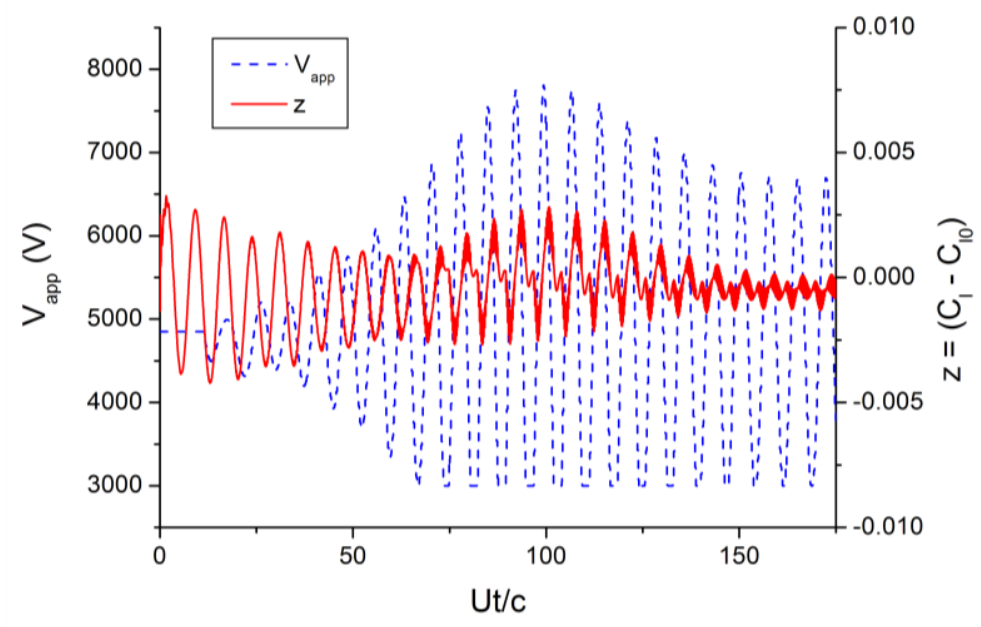

a) Time history of performance and control output

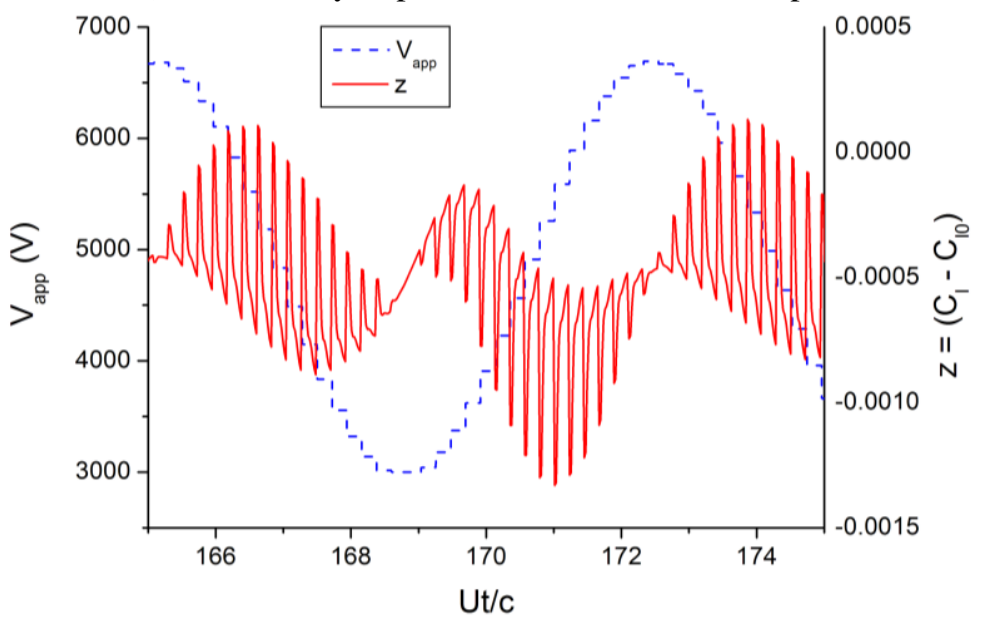

b) Enlarged time history

Figure 13. Adaptive control for rejecting the disturbance in inlet flow speed $\left(\alpha=2^{\circ}, V_{\text {app }}=3-8 \mathrm{kV}\right)$.

The remaining fluctuation even with control is partially due to the updating/sampling time step size of the controller. Since the control output and the performance measurement are done every $10^{\text {th }}$ time step of the flow solver, the performance spikes in Figure $13 \mathrm{~b}$ ), which corresponds to the system response to the step voltage variation, are ignored in the controller. The fluctuation of the nominal performance variation sampled with $\Delta \mathrm{t}_{\mathrm{c}}$, which is the variation without the spikes, is about $10 \%$ of the uncontrolled value.

For the case with $\alpha=8^{\circ}$ the corresponding time history is presented in Figure 14 and the feedback controller is activated at $\mathrm{Ut} / \mathrm{c}=17.8$. Compared to Figure 13 , which involves no separation bubble, the response of the performance to the voltage exhibits more irregular evolution, implying increased nonlinearity as expected. Although the impact of the inlet disturbance exists until $\mathrm{Ut} / \mathrm{c}=150$, the performance offset is successfully eliminated and the performance fluctuation amplitude is decreased to $40 \%$ of the uncontrolled value. Since the change in voltage amplitude between time steps is much lower than the previous $\alpha=2^{\circ}$ case, no noticeable performance peaks are observed.

In order to assess the transient time for accomplishing performance minimization and enhance the transient response, further study is necessary. The prescribed voltage limits of the input to the DBD actuator affect control authority and may induce additional nonlinearity to the system. At the same time, a proper updating/sampling time 
step size corresponding to the disturbance time scale needs to be investigated to avoid the unresolved performance peaks and improve control efficiency.

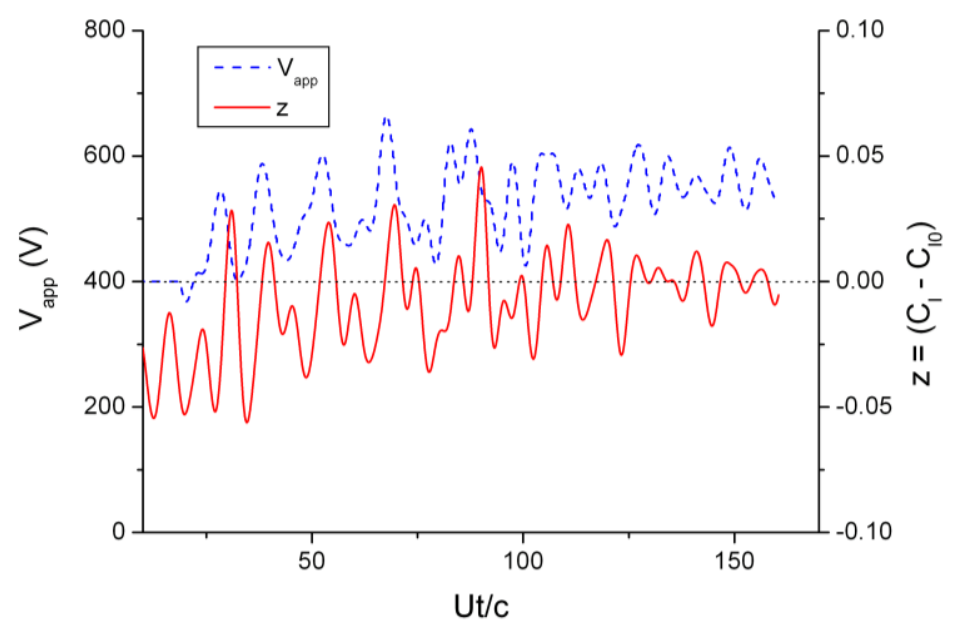

Figure 14. Adaptive control for rejecting the disturbance in inlet flow speed $\left(\alpha=8^{\circ}, V_{\text {app }}=0.1-0.7 \mathrm{kV}\right)$.

\section{Conclusion}

Due to the light weight and slow flight speed, a low Reynolds number flyer is inherently susceptible to uncertainties of the flow environment. In this preliminary study, using a recently developed ARMARKOV/Toeplitz control scheme, the characteristics of the adaptive control in response to the fluctuation of the free stream and the corresponding flow structures around a SD7003 airfoil at the chord Reynolds number of $6 \times 10^{4}$ are investigated. The unsteady performance evolution and related vortex evolution mechanisms, depending on the actuation mode, are presented, and relatively simple cases with low angle of attack are chosen to apply feedback control. The reduced order plasma model is used to simulate the DBD actuator on the low Reynolds number airfoil, and an adaptive control scheme based on the $\mu$-Markov model is applied. Combined with the off-line time-domain system identification, the adaptive algorithm successfully regulates the performance of the airfoil such as command following and disturbance rejection. The recursive least squares method is used to estimate the system parameters, which are in agreement with the impulse response.

As the angle of attack increases the flow structure is dominated by vortex formation, convection and detachment in the wake region. Various factors affecting the control authority such as actuator position, operating voltage range and number of actuators need to be studied accordingly. In order to cope with the change in flow dynamics, on-line system identification and a strategy for efficient measurement can be studied further.

\section{Acknowledgement}

This work has been supported in part by the Michigan/AFRL (Air Force Research Laboratory)/Boeing Collaborative Center in Aeronautical Sciences. 
1 Shyy, W., Lian, Y., Tang, J., Viieru, D., and Liu, H., Aerodynamics of Low Reynolds Number Flyers, Cambridge Univ. Press, New York, 2008

2 Lissaman, P. B. S., "Low-Reynolds-number airfoils," Ann. Rev. Fluid Mech. Vol. 15, 1983, pp.223-239

3 Mueller, T. J., and Delaurier, J. D., “Aerodynamics of small vehicles," Ann. Rev. Fluid Mech., Vol. 35, 2003, pp.89-111

4 Tani, I., "Low-speed flows involving bubble separations," Prog. Aero. Sci., Vol. 5, 1964, pp.70-103

5 Jayaraman, B., Lian, Y., and Shyy, W., "Low-Reynolds number flow control using dielectric barrier discharge actuators," AIAA Paper 2007-3974, 37 ${ }^{\text {th }}$ AIAA Fluid Dynamics Conference and Exhibit, 25-28 June, Miami, FL, 2007

6 Visbal, M. R., Gaitonde, D. V., and Roy, S., "Control of transitional and turbulent flows using plasma-based actuators," AIAA Paper 2006-3230, 36 th AIAA Fluid Dynamics Conference and Exhibit, 5-8 June, San Francisco, CA, 2006

7 Moreau, E., "Airflow control by non-thermal plasma actuators," J. Phys. D: Appl. Phys. 40, 2007, pp.605-636

8 McDaniel, E. W., Collision Phenomena in Ionized Gases, John Wiley \& Sons, Inc., New York, 1964

9 Boeuf, J. P., "Numerical model of rf glow discharges," Phys. Rev. A 36 (6), 1987, pp. 2782-2792

${ }^{10}$ Roth, J. R., and Sherman, D. M., "Boundary layer flow control with a one atmosphere uniform glow discharge surface plasma," AIAA Paper 98-0328, 1998

${ }^{11}$ Jayaraman, B., Cho, Y., and Shyy, W., "Modeling of dielectric barrier discharge plasma actuator," J. Appl. Phys., 103, 053304, 2008

${ }^{12}$ Enloe, C. L., McLaughlin, T. E., VanDyken, R. D., Kachner, K. D., Jumper, E. J., and Corke, T. C., "Mechanisms and responses of a single dielectric barrier plasma actuator: plasma morphology," AIAA Journal, Vol. 42, No. 3, March 2004, pp. 589-594

${ }^{13}$ Pons, J, Moreau, E. and Touchard, G., "Asymmetric surface dielectric barrier discharge in air at atmospheric pressure: electrical properties and induced airflow characteristics," J. Phys. D: Appl. Phys., Vol. 38, 2005, pp.3635-3642

${ }^{14}$ Jayaraman, B., and Shyy, W., "Modeling of dielectric barrier discharge-induced fluid dynamics and heat transfer," Prog. Aero. Sci., doi: 10.1016/i.paerosci.2007.10.004, 2007

${ }^{15}$ Shyy, W., Jayaraman, B., and Anderson, A., "Modeling of glow-discharge induced flow dynamics," J. Appl. Phys., 92(11), 2002, pp.6434-6443

${ }^{16}$ Visbal, M. R., Gaitonde, D. V., and Roy, S., "Control of transitional and turbulent flows using plasma-based actuators," AIAA Paper 2006-3230, 36 ${ }^{\text {th }}$ AIAA Fluid Dynamics Conference and Exhibit, 5-8 June, San Francisco, CA, 2006

${ }^{17}$ Rizzetta, D. P., and Visbal, M. R., "Numerical investigation of plasma-based flow control for transitional highly loaded low-pressure turbine," AIAA Journal, Vol. 45, No. 10, October 2007, pp.2554-2564

${ }^{18}$ Greenblatt, D., Goeksel, B., Schule, C. Y., and Paschereit, C. O., "Dielectric barrier discharge flow control at very low flight Reynolds numbers," 47 $7^{\text {th }}$ Israel Annual Conference on Aerospace Sciences, 21-22 February, Haifa, 2007

${ }^{19}$ Asghar, A., and Jumper, E. J., "Phase synchronization of vortex shedding from multiple cylinders using plasma actuators," AIAA Paper 2003-1028, 41 ${ }^{\text {st }}$ AIAA Aerospace Sciences Meeting and Exhibit, 6-9 January, Reno, NV, 2003

${ }^{20}$ Huang, X., Chan, S., Zhang, X. and Gabriel, S., "Variable structure model for flow-induced tonal noise control with plasma actuators," AIAA Journal, Vol. 46, No. 1, January 2008, pp.241-250

${ }^{21}$ Lian, Y. and Shyy, W., "Laminar-turbulent transition of a low Reynolds number rigid or flexible airfoil," AIAA Journal, Vol. 45, No. 7, July 2007, pp. 1501-1513

${ }^{22}$ Kutay, A. T., Calise, A. J. and Muse, J. A., "A 1-DOF wind tunnel experiment in adaptive flow control," AIAA Paper 2006-6430, AIAA Guidance, Navigation and Control Conference and Exhibit, 21-24 August, Keystone, CO, 2006

${ }^{23}$ Rowley, C. W., Ahuja, S., Taira, K. and Colonius, T., "Closed-loop control of leading edge vorticity on a 3D wing: simulations and low-dimensional models," AIAA Paper 2008-3981, 38 ${ }^{\text {th }}$ Fluid Dynamics Conference and Exhibit, 23-26 June, Seattle, WA, 2008

${ }^{24}$ Venugopal, R., and Bernstein, D. S., "Adaptive disturbance rejection using ARMARKOV/Toeplitz models," IEEE Trans. Contr. Sys. Tech., Vol. 8, No. 2, 2000, pp. 257-269

25 Åström, K. J., and WittenMark, B., Adaptive Control, $2^{\text {nd }}$ ed., Addison-Wesley Publishing Company Inc., New York, 1995

${ }^{26}$ Pindera, M. Z., "Adaptive flow control using simple artificial neural networks," AIAA Paper 2002-0990, 40 ${ }^{\text {th }}$ AIAA Aerospace Sciences Meeting and Exhibit, 14-17 January, Reno, 2002

${ }^{27}$ Santillo, M. A., Hoagg, J. B., Bernstein, D. S., and Powell, K., "CFD-based adaptive flow control for steady flow field modification," Proceedings of the $45^{\text {th }}$ IEEE Conference on Decision and Control, 13-15 December, San Diego, 2006

${ }^{28}$ Tian, Y., Song, Q., and Cattafesta, L., "Adaptive feedback control of flow separation," AIAA Paper 2006-3016, $3^{\text {rd }}$ AIAA Flow Control Conference, 5-8 June, San Francisco, CA, 2006

${ }^{29}$ Grundmann, S., Klumpp, S. and Tropea, C., Experimental and numerical investigations of boundary-layer influence using plasma-actuators. In: R. King, ed. Active Flow Control, NNFM 95, New York: Springer, 2007, pp. 56-68

${ }^{30}$ Akers, J. C. and Bernstein, D. S., "Time-domain identification using ARMARKOV/Toeplitz Models," Proceedings of the American Control Conference, Albuquerque, New Mexico, June 1997

${ }^{31}$ Juang, J.-N., Phan, M., Horta, L., and Longman, R., "Identification of observer/Kalman filter Markov parameters: theory and experiments," NASA TM-104069, March 2000

${ }^{32}$ Ljung, L. and Söderström T., Theory and Practice of Recursive Identification, The MIT Press, Cambridge, Massachusetts, 1983

${ }^{33}$ Sayed, A. H., Adaptive Filters, John Wiley \& Sons, Inc., Hoboken, New Jersey, 2008 